\title{
Guest Editorial Foreword to the Special Issue of IHC 2017
}

The Brazilian Symposium on Human Factors in Computing Systems (IHC) is the major scientific event in HumanComputer Interaction in Latin America. Yearly, IHC brings together academics, practitioners and students interested in scientific investigation on theory and practices related to the design and use of interactive systems. Together, the participants debate methods, tools and experiences related to conceiving, creating, and evaluating interactive computing solutions to be used by people, as well as the major social phenomena surrounding the technology adoption and appropriation. Traditionally, IHC has been an important forum for dissemination of innovative ideas and practical solutions for everyday issues, but also about ethics and social aspects of those involved in technology research and design, as well as professional education and critical thinking.

Held in Joinville city, IHC had its 16th edition embracing the theme "Emotion and Movement". With Emotion and Movement, IHC highlighted the influence of emotional and affective aspects on designing and using of computing solutions. This thematic brought to the center of the debate human aspects that pervade research and practice of Human Computer Interaction and are relevant to Computing in a comprehensive way.

IHC 2017 received 184 valid submissions, from which 51 (28\%) were accepted as Full Papers. This Special Issue of the SBC Journal on Interactive Systems includes the 7 best full papers from IHC 2017, in revised and extended versions. The first paper presents a theoretical discussion and a methodological approach for understanding and designing in the context of sociotechnical scenarios, combining the SociallyAware Design approach with the Actor-Network Theory. The second paper focuses on accessibility issues in mobile apps, analyzing the adequacy of interface components for screen readers, and providing insights for research and practice in this domain. The third paper contributes to investigations in Big Data from HCI perspective. The authors propose, exemplify and evaluate a strategy for measuring learning curves in large datasets containing information about how users performed real tasks. The fourth paper tackles the challenge of representing quality in models used in the design of interactive systems by investigating how experienced and inexperienced designers use and evaluate a usability-oriented interaction and navigation model, which was created to express usability mechanisms in interaction and navigation modeling solutions. By exploring user interaction with chatbots, the fifth paper applies the Semiotic Inspection Method to investigate designers' strategies for conveying chatbot features to users, and identifies and exemplifies common strategies designed in existing chatbots, offering critical discussions and challenges for designers. The sixth paper brings inclusion and ethics into perspective, presenting a review on LGBT issues in HCI literature and mobile applications and describing a semio-participatory approach for the codesign of applications, discussing how to address ethics and social concerns in product design. The seventh paper describes and evaluates a visualization-based interface of a biodiversity information system with findings on how to explore and evaluate visualization techniques for interacting with large datasets.

The seven extended papers cover topics from data visualization and analysis to system modelling and evaluating techniques, presenting methodological and theoretical contributions in both applied and academic contexts, and recognizing technical, formal and social issues in HCI research and development. The editors thank the authors and the reviewers for the careful and competent work, and invite the readers to enjoy and get inspired by this selection of the best of the Brazilian HCI research in 2017.

\author{
Isabela Gasparini (UDESC) \\ Lara Piccolo (The Open University) \\ Luciana Salgado (UFF) \\ Luciana Zaina (UFSCar) \\ Roberto Pereira (UFPR)
}

